\title{
Genetic variability in Acidithiobacillus spp. - a working horse of environmental biotechnologies
}

\author{
Peter Pristas ${ }^{1, \bigotimes}$, Jana Kiskova ${ }^{1}$, Ivana Timkova ${ }^{1}$, Lenka Malinicova ${ }^{1}$, Alena Luptakova ${ }^{2}$, \\ Maria Kusnierova $^{2}$ and Jana Sedlakova-Kadukova ${ }^{1}$
}

${ }^{1}$ Department of Microbiology, Institute of Biology and Ecology, Faculty of Science, Pavol Jozsef Šafarik University in Košice, Šrobárova 2, Košice, 041 54, Slovakia

${ }^{2}$ Institute of Geotechnics, Slovak Academy of Sciences, Watsonova 45, Kořice, 043 53, Slovakia

\section{Article info}

Article history:

Received: $16^{\text {th }}$ October 2018

Accepted: $21^{\text {st }}$ December 2018

\section{Keywords:}

Acidithiobacillus

Complex species

Environmental biotechnology

Genetic variability

Phylogeography

\begin{abstract}
The genus Acidithiobacillus comprises 7 species of Gram-negative obligatory acidophilic chemolithotrophic bacteria that derive energy mainly from the oxidation of reduced sulphur compounds. Four of the species also catalyse the dissimilatory oxidation of ferrous iron while three (A. thiooxidans, A. albertensis, and A. caldus) do not. Bacteria from the genus Acidithiobacillus are often associated with mineral biotechnologies (biomining) and acid mine drainage. While acceleration of mineral solubilisation is a positive aspect in environmental biotechnologies, it is undesirable in acid mine drainage with strong negative ecological impact and there is profound interest in genetics and genomics of these bacteria. Representatives of Acidithiobacillus genus occur world-wide, however there are limited data on Acidithiobacillus spp. variability from Slovakia. In our work the variability of Acidithiobacillus spp., from Slovakia was analysed and the presence of A ferrooxidans was detected. In addition, for the first time we report here on the occurrence of $A$. albertensis as well. Comparative analyses confirmed pronounced genetic and genomic diversity within the genus, especially within A. ferrooxidans and A. thioxidans complexes. Based on data presented, several Acidithiobacillus species could be considered as a complex species and the description of several new species is very probable in the near future.
\end{abstract}

\section{Introduction}

The genus Acidithiobacillus comprises a group of Gram-negative obligatory acidophilic chemolithotrophic bacteria that derive energy mainly from the oxidation of reduced sulphur compounds. The members of the genus are motile by one or more flagella and comprise both mesophiles and moderate thermophiles (Kelly and Wood 2000). Acidithiobacilli use various sulphur compounds as electron donor and therefore, are often found in metal sulphide deposits all over the world (Karavaiko et al. 2003), fresh waters associated with sulphide deposits (Gonzalez-Toril et al. 2003) and seawater (Kamimura et al. 2003). The final electron acceptor is usually oxygen (Kelly and Wood 2000) however, $\mathrm{Fe}^{3+}$ and $\mathrm{S}^{0}$ were reported to be alternative electron acceptors for some species (Ohmura et al. 2002). The ability to fix $\mathrm{CO}_{2}$ makes acidithiobacilli independent of an organic carbon source and allows them to thrive in mine waste ecosystems where the organic carbon concentration is extremely low (Dold et al. 2005).

The Acidithiobacillus genus was formed when the former Thiobacillus genus was split into the genera 
Acidithiobacillus, Halothiobacillus, and Thermithiobacillus (Kelly and Wood 2000). The type species of the genus, A. thiooxidans was described nearly 100 years ago as Thiobacillus thiooxydans (Waksmann and Joffe 1922) and up to now 7 species within the genus were validly described. All species are autotrophs capable of growth utilising inorganic sulphur compounds as sole energy substrate. Four of the species (A. ferriphilus, A. ferrivorans, A. ferrooxidans, and A. ferridurans) also catalyse the dissimilatory oxidation of ferrous iron while three (A. thiooxidans, A. albertensis, and A. caldus) do not. Representatives of Acidithiobacillus genus occur world-wide in a diverse range of natural (acid rock drainage, sulphur springs, etc.) and industrial settings (ore concentrates, leaching solutions of the mining industry, etc.), with varying physicochemical characteristics.

Bacteria from the genus Acidithiobacillus are often associated with mineral biotechnologies (biomining) and acid mine drainage. Biomining utilises these bacteria for recovery of metals from sulphidic low grade ores and concentrates. Acid mine drainage results in acidification and metal contamination of soil and water emanating from the dissolution of metal sulphides from deposits and mine waste storage (Johnson 2014). Acidophilic microorganisms play a central role in these processes by catalysing aerobic oxidation of sulphides. While acceleration of mineral solubilisation is a positive aspect in mineral biotechnologies, it is undesirable in acid mine drainage with strong negative ecological impact and there is profound interest in genetics and genomics of these bacteria.

DNA based analyses have already revealed pronounced genetic diversity within Acidithiobacillus genus, especially within A. ferrooxidans complex. The diversity probably could be not attributed to the geographic distribution of acidithiobacilli as practically all known species were detected worldwide (Nunez et al. 2017). More than $50 \%$ of all Acidithiobacillus isolates characterised at $16 \mathrm{~S}$ sequence level can be mapped to Asia, followed by Europe $(20 \%)$, South (9\%), and North America (7\%), and Africa and Oceania (less than $2 \%$ each). From the Slovakia territory two isolates were characterised, both identified as A. ferrooxidans
(Nunez et al. 2017). The aim of our study was to understand the variability within acidithiobacilli in Slovakia and worldwide based on genetic and genomic analyses.

\section{Experimental}

\section{Molecular identification of Acidithiobacillus spp. isolates from the Slovakia}

Molecular identification of Acidithiobacillus spp. isolated recently from Smolnik (East Slovakia) acid mine drainage water - LC isolate (Luptakova et al. 2012) and A. albertensis TS isolate from HodrušaHámre (Central Slovakia) gold mine (Pristas et al.; manuscript in preparation) were performed using widely accepted 16S based methodology (Yarza et al. 2014). The sequences obtained were deposited into GenBank database under accession numbers MH740926 and MH796351 for LC and TS isolate, respectively. The sequences were compared against database of $16 \mathrm{~S}$ sequences of type strains of bacteria and archaea using BLASTN analysis (Altschul et al. 1990). Phylogenetic placement of isolates was confirmed by multiple sequence comparison against 528 type strains of Acidithiobacillus spp. downloaded from RDP database (available at https://rdp.cme.msu.edu/) using the NeighborJoining method implemented in MEGA 7 software (Kumar et al. 2016) with 500 bootstrap replications.

\section{Analysis of genetic variability within Acidithiobacillus genus}

To analyse genetic variability in Acidithiobacillus genus 16S rRNA sequences of LC and TS isolates obtained in this study were added to the pre-aligned 16S Acidithiobacillus spp. sequences and analysed as described above. For genome comparison, available genome sequences of Acidithiobacillus spp. were downloaded from the GenBank database (https://www.ncbi.nlm.nih.gov/genome). Final dataset was composed of 8 sequences GCA_001931655 for A. albertensis, GCA_000214095.3 for A. ferrivorans, GCA_000175575.2 for A. caldus, GCA_000227215.2 for A. thiooxidans, 
GCA_000021485 for A. ferrooxidans, GCA_000179815.2 for Acidithiobacillus sp. GGI221, GCA_002341825.1 for Acidithiobacillus sp. UBA2486, and GCA_002733395.1 for Acidithiobacillus sp. NORP59. For each pair of sequences ANI value (Average Nucleotide Identity) was calculated using EzTaxon server (https://www.ezbiocloud.net/tools/ani; Yoon et al. 2017). Based on ANI values obtained a similarity dendrogram was constructed using UPGMA algorithm implemented in MEGA 7. For functional genome comparisons the RAST server pipeline (Rapid Annotation by Subsystem Technology) available at https://rast.theseed.org/ was used (Overbeek et al. 2014).

\section{Results and Discussion}

Two recently isolated Acidithiobacillus spp. isolates were identified using $16 \mathrm{~S}$ rRNA sequencing followed by BLASTN analysis of sequences obtained. The 16S rRNA sequence of LC isolate from Smolnik acid mine drainage water showed the highest similarity $(99.7 \%)$ to the $16 \mathrm{~S}$ rRNA sequences of type species of A. ferrooxidans strain ATCC 23270. The 16S rRNA sequence of TS isolate from Hodruša-Hámre gold mine has shown the highest similarity (99.7 \%) to the A. albertensis DSM 14366 sequence. Multiple sequence alignments confirmed identification of both isolates resulted from BLASTN analysis (Fig.1). The LC isolate sequence grouped with $A$. ferrooxidans sequence, while this of TS isolate grouped with $A$. albertensis sequence.
The tree indicates that the ability to obtain energy from the dissimilatory oxidation of ferrous iron is probably of old evolutionary origin as all four species able to utilise ferrous ions (A. ferriphilus, A. ferrivorans, A. ferrooxidans, and A. ferridurans) formed well supported group phylogenetically distant from the species unable to utilize ferrous ions. The analysis indicates that $A$. caldus is the most distant species of Acidithiobacillus genus, forming a separate branch non-related to the other members of the genus (Fig. 1).

Up to now, up to 120 Acidithiobacillus spp. isolates or environmental 16S rRNA sequences were identified from the European territory, most of them identified as A. ferrooxidans and A. thiooxidans and the occurrence of A. ferrooxidans related bacteria have already been reported from Slovakia as well (Nunez et al. 2017). However, no A. albertensis related sequences have been reported neither from the Slovak nor European territory yet. A. albertensis species was described in 1983 (Bryant et al. 1983) and it is not studied very frequently. A. albertensis related isolates were reported up to now mainly from China and Canada (Nunez et al. 2017), while our observation is the first report on A. albertensis from Europe.

Biotechnology and environmental protection interest led to the characterization of multiple Acidithiobacillus spp. isolates mainly from industrial environments. Most of the isolates were identified as $A$. ferrooxidans or A. thiooxidans as for many years these two species were the only validly described species of the Acidithiobacillus

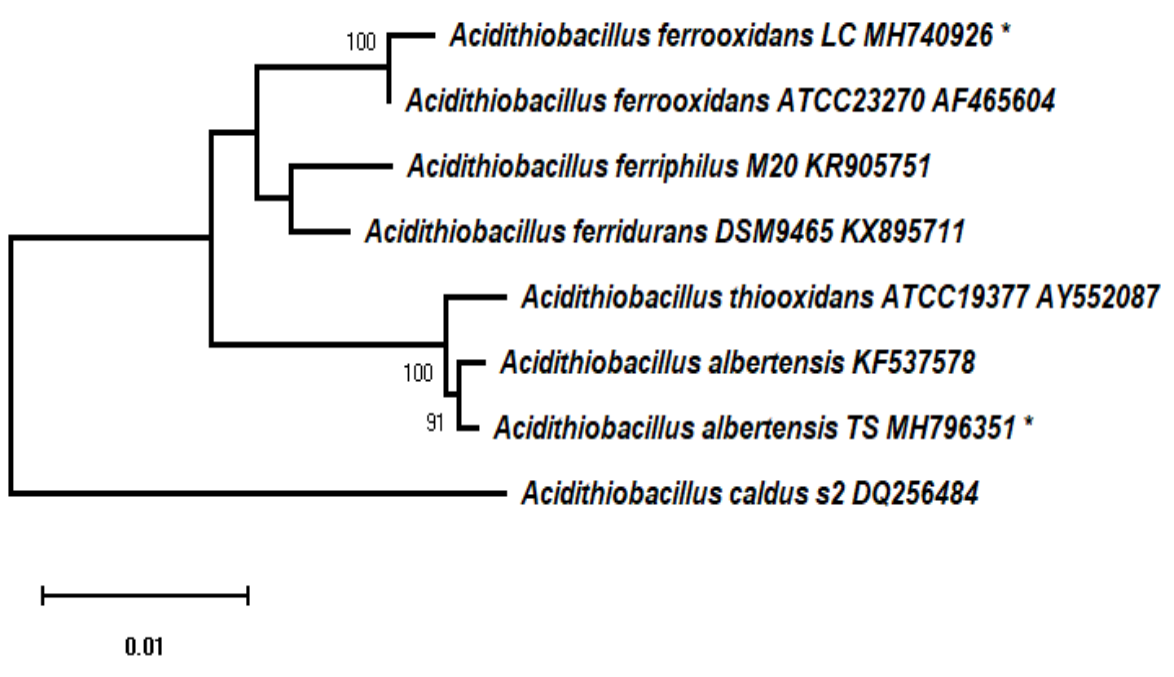

Fig. 1. Unrooted phylogenetic tree documenting phylogenetic placement of Acidithiobacillus spp. from Slovakia (A. ferrooxidans LC and A. albertensis $\mathrm{TS}$; indicated with asterisks) inferred using the Neighbor-Joining method. The bar represents genetic distance shown in number of base substitutions per site. Numbers at nodes are bootstrap values after 500 repetitions. 


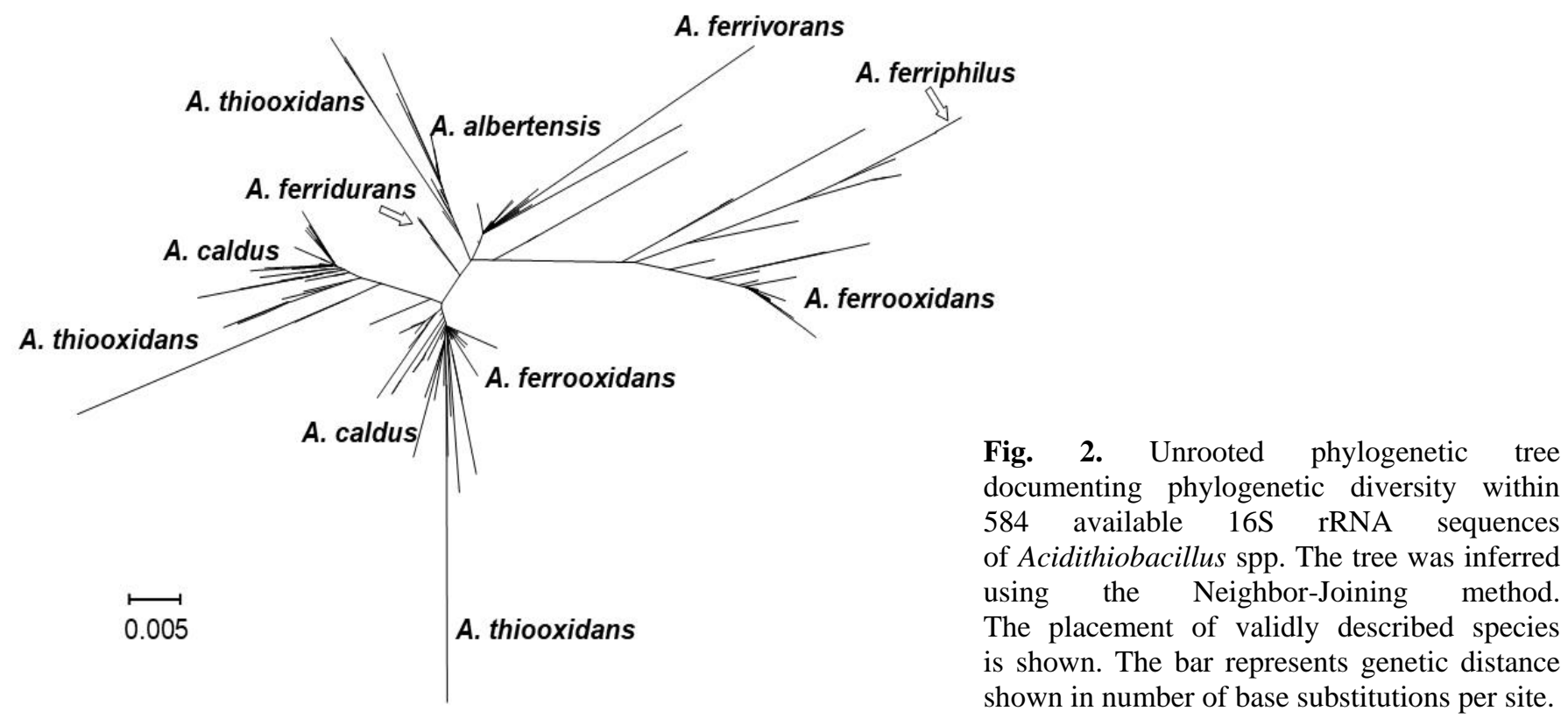

genus (Jones et al. 2016). In the recent years great diversity within the genus was recognised and several new species of acidithiobacilli were described (Hallberg et al. 2010; Hedrich and Johnson 2013; Falagan and Johnson 2016). To understand genetic variability in Acidithiobacillus genus all available 16S rRNA sequence of Acidithiobacillus-related bacteria were downloaded from RDP database (Cole et al. 2014) and compared using phylogenetic approach. The resulted phylogenetic tree (Fig. 2) clearly documents extraordinary variability within Acidithiobacillus genus. While the recently described species e.g. A. ferrivorans and A. ferridurans form monophyletic, well supported clades in the tree, other species are spread over the tree and could be found in multiple branches, indicating genetic variability within the genus. The diversity within Acidithiobacillus genus have already been reported (Karavaiko et al. 2003; Ni et al. 2008) and based on our data the existence of at least 20 species could be proposed solely on 16s rRNA analysis.

Recent advances in genomics allowed us to better understand the common and distinctive features some of Acidithiobacillus species isolated from similar habitats and provided data to understand potential adaptation mechanisms of these bacteria to the harsh environments. Complete or draft genome sequences of several Acidithiobacillus species have become available in the recent few years (Cárdenas et al. 2016). Comparative genomic analyses indicate that genomes of acidithiobacilli are relatively similar. The genome sizes vary from 2.78 to $3.78 \mathrm{Mbp}, \mathrm{G}+\mathrm{C}$ content vary from 52.5 to $67.5 \%$, and numbers of predicted genes vary from 2648 to 4007 . In all these parameters A. caldus is the most distant species, showing the highest $\mathrm{G}+\mathrm{C}$ content, the smallest genome size, and the smallest number of genes (data not shown). Based on the functional comparative genomic analyses of Acidithiobacillus species, it was shown that different metabolic pathways might allow these microorganisms to acquire energy, carbon sources, and nitrogen sources from acidic environments, ensuring their survival and proliferation in these environments (Fig. 3). Using RAST server pipeline (Overbeek et al. 2014) very similar numbers of genes were found e.g. those of involved in RNA metabolism or cell wall and capsule subsystems in all Acidithiobacillus species analysed. The species, however, differ significantly in number of genes classified to the Nitrogen or Phosphorus metabolism, and Protein metabolism, as well as stress response (Fig. 3). The comparisons indicate that non-motile species of the genus (A. ferrooxidans and A. thioxidans) completely lack the genes for motility and chemotaxis. The phenotypic diversity observed within the genus is thus a consequence of genetic and genomic diversity.

To evaluate the genomic diversity in more detail, 


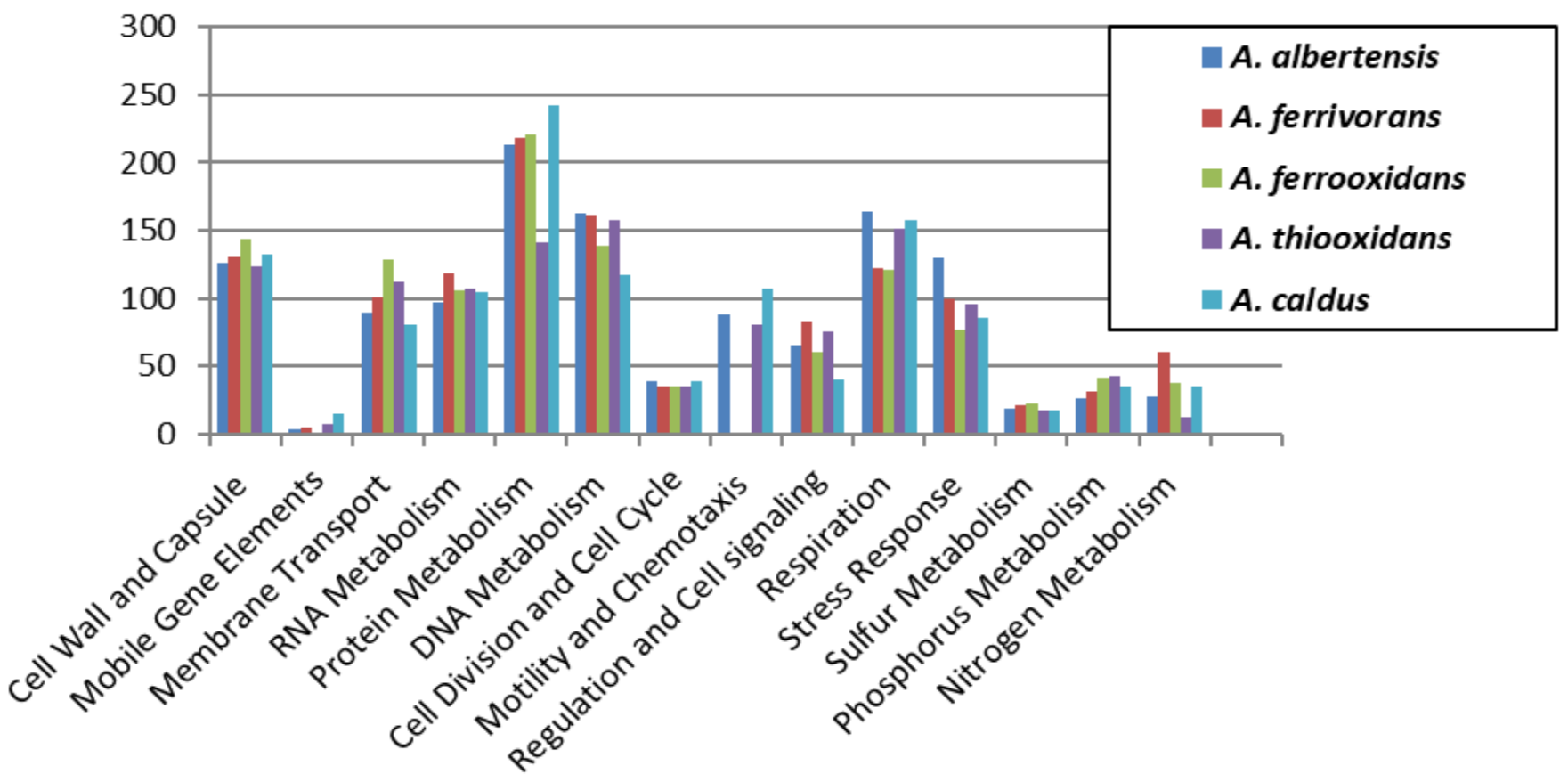

Fig. 3. Comparative genomics of selected Acidithiobacillus species. Number of genes classified to the selected subsystems as identified by RAST server is shown.

average nucleotide identity (ANI) values were calculated for every pair of genomic sequences and compared. ANI was proposed recently as a means to compare genetic relatedness among prokaryotic strains. The method compares nucleotide-level genomic similarity between the coding regions of two genomes and is considered the best measure of genetic relatedness among bacterial strains (Figueras et al. 2014; Jain et al. 2018). ANI values in range from 0.993 to 0.640 were observed among analysed set of sequences. Based on ANI values relatedness tree of Acidithiobacillus spp. was constructed (Fig. 4). The tree shows congruent topology with 16S rRNA based tree (Fig. 1).
The ferrous iron-oxidising species form a separate group distant from the species that are unable to utilize ferrous ions, and (like in $16 \mathrm{~S}$ rRNA based tree) A. caldus is the most divergent species of Acidithiobacillus genus related distantly only to any other species. The tree also documents diversity within the genus when the yet uncharacterised isolates UBA2486 and NORP59 have produced significantly low ANI values and therefore could be assigned as a new species within Acidithiobacillus genus. Similarity values higher than 0.95 are necessary to place the isolates to the same species (Figueras et al. 2014).

The first species of the Acidithiobacillus genus was
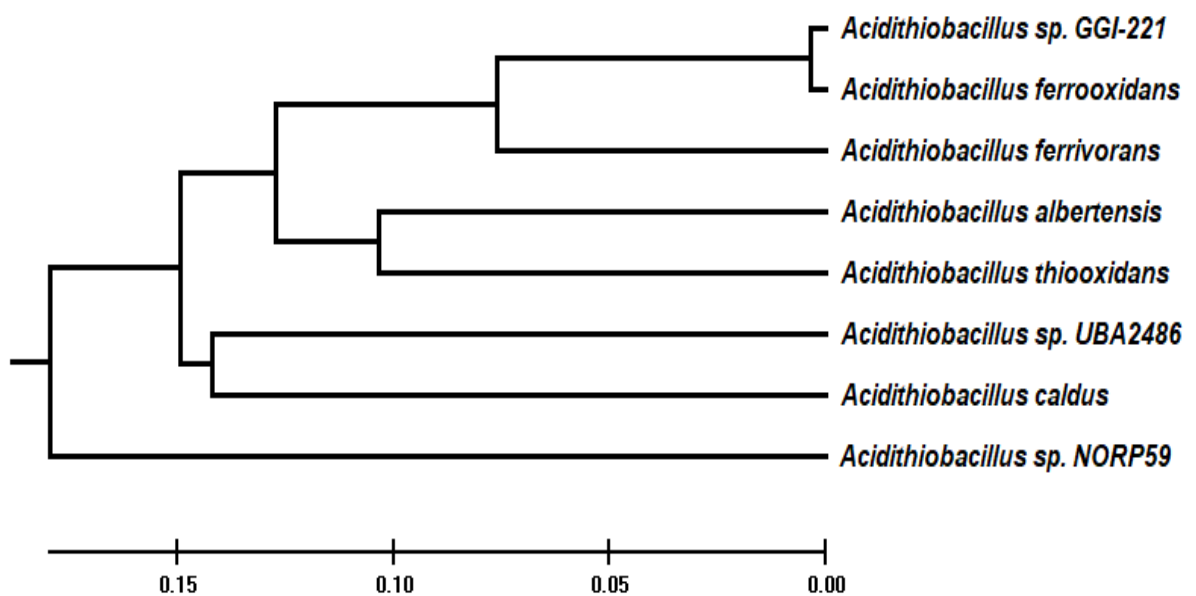

Fig. 4. UPGMA (unweighted pair group method with arithmetic mean) generated dendrogram showing relatedness of Acidithiobacillus sp. based on ANI (average nucleotide identity) values. 
described already 100 years ago (Waksmann and Joffe 1922) and up to now 7 species of acidithiobacilli were validly described, most of them in the recent 20 years. However, application of modern genetic and genomic methods indicates that variability within the genus is highly underestimated and several species, especially A. ferrooxidans and A. thioxidans, have to be considered a complex species and the description of multiple new species of acidithiobacilli is very probable in the near future.

\section{Conclusions}

Our data indicate that genetic variability of Acidithiobacillus genus is highly underestimated. Several species, especially A. ferrooxidans and $A$. thioxidans, have to be considered a complex species and the description of multiple new species of acidithiobacilli is very probable in the near future. From the Slovakia territory A. ferrooxidans species is reported predominantly, but for the first time we report here on the occurrence of $A$. albertensis as well.

\section{Acknowledgement}

The work was financially supported by Research Agency of the Ministry of Education, Science, Research and Sport of Slovak Republic under the VEGA Project 1/0229/17.

\section{References}

Altschul SF, Gish W, Miller W, Myers EW, Lipman DJ (1990) Basic local alignment search tool. J. Mol. Biol. 215: 403-410.

Bryant RD, McGroarty KM, Costerton JW, Laishley EJ (1983) Isolation and characterization of a new acidophilic Thiobacillus species (T. albertis). Can. J. Microbiol. 29: 1159-1170.

Cárdenas JP, Quatrini R, Holmes DS (2016) Progress in acidophile genomics. In Quatrini R, Johnson DB (Eds.), Acidophiles: life in extremely acidic environments, Caister Academic Press, UK, pp. 179198.

Cole JR, Wang Q, Fish JA, Chai B, McGarrell DM, Sun Y, Brown CT, Porras-Alfaro A, Kuske CR, Tiedje TM (2014). Ribosomal Database Project: data and tools for high throughput rRNA analysis Nucl. Acids Res. 42 (Database issue): D633-D642.

Dold B, Blowes DW, Dickhout R, Spangenberg JE, Pfeifer HR (2005). Low molecular weight carboxylic acids in oxidizing porphyry copper tailings. Environ. Sci. Technol. 39: 2515-2521.

Hallberg KB, González-Toril E, Johnson DB (2010) Acidithiobacillus ferrivorans sp. nov.; facultatively anaerobic, psychrotolerant iron-, and sulfur-oxidizing acidophiles isolated from metal mine-impacted environments. Extremophiles 14: 9-19.

Hedrich S, Johnson DB (2013) Acidithiobacillus ferridurans sp. nov., an acidophilic iron-, sulfur- and hydrogenmetabolizing chemolithotrophic gammaproteobacterium. Int. J. Syst. Evol. Microbiol. 63: 4018-4025.

Falagan C, Johnson DB (2016) Acidithiobacillus ferriphilus sp. nov., a facultatively anaerobic iron- and sulfurmetabolizing extreme acidophile. Int. J. Syst. Evol. Microbiol. 66: 206-211.

Figueras MJ, Beaz-Hidalgo R, Hossain MJ, Liles MR (2014) Taxonomic affiliation of new genomes should be verified using average nucleotide identity and multilocus phylogenetic analysis. Genome Announc. 2: e00927-14.

Gonzalez-Toril E, Llobet-Brossa E, Casamayor EO, Amann R, Amils R (2003) Microbial ecology of an extreme acidic environment, the Tinto River. Appl. Environ. Microbiol. 69: 4853-4865.

Jain C, Rodriguez RLM, Phillippy AM, Konstantinidis KT, Aluru S (2018) High throughput ANI analysis of 90K prokaryotic genomes reveals clear species boundaries. Nature Comm. 9: Article number: 5114.

Johnson DB (2014) Biomining-biotechnologies for extracting and recovering metals from ores and waste materials. Curr. Opin. Biotechnol. 30: 24-31.

Jones DS, Schaperdoth I, Macalady JL (2016) Biogeography of sulfur-oxidizing Acidithiobacillus populations in extremely acidic cave biofilms. The ISME Journal 10: 2879-2891.

Kamimura K, Higashino E, Moriya S, Sugio T (2003). Marine acidophilic sulfur oxidizing bacterium requiring salts for the oxidation of reduced inorganic sulfur compounds. Extremophiles 7: 95-99.

Karavaiko GI, Turova TP, Kondraeva TF, Lysenko AM, Kolganova TV, Ageeva SN, Muntyan LN, Pivovarova TA (2003) Phylogenetic heterogeneity of the species Acidithiobacillus ferrooxidans. Int. J. Syst. Evol. Microbiol. 53: 113-119.

Kelly DP, Wood AP (2000) Reclassification of some species of Thiobacillus to the newly designated genera Acidithiobacillus gen. nov., Halothiobacillus gen. nov. and Thermithiobacillus gen. nov. Int. J. Syst. Evol. Microbiol. 50: 511-516.

Kumar S, Stecher G, Tamura K (2016) MEGA7: Molecular Evolutionary Genetics Analysis Version 7.0 for Bigger Datasets. Mol. Biol. Evol. 33: 1870-1874.

Luptakova A, Prascakova M, Kotulicova I (2012) Occurrence of Acidithiobacillus Ferrooxidans bacteria in sulfide mineral deposits of Slovak Republic. Chem. Eng. Trans. 28: 31-36.

Ni YQ, He KY, Bao, JT, Yang Y, Wan DS, Li HY (2008) Genomic and phenotypic heterogeneity of Acidithiobacillus spp. strains isolated from diverse habitats in China FEMS Microbiol. Let. 64: 248-259. 
Nunez H, Moya-Beltran A, Covarrubias PC, Issotta F, Cardenas JP, Gonzalez M, Atavales J, Acuna LG, Johnson DB, Quatrini R (2017) Molecular systematics of the genus Acidithiobacillus: insights into the phylogenetic structure and diversification of the taxon. Front. Microbiol. 8: 30.

Ohmura N, Sasaki K, Matsumoto N, Saiki H (2002). Anaerobic respiration using $\mathrm{Fe}^{3+}, \quad \mathrm{S}^{0}$ and $\mathrm{H}_{2}$ in the chemolithotrophic bacterium Acidithiobacillus ferrooxidans. J. Bacteriol. 184: 2081-2087.

Overbeek R, Olson R, Pusch GD, Olsen GJ, Davis JJ, Disz T, Edwards RA, Gerdes S, Parrello B, Shukla M, Vonstein V, Wattam AR, Xia F, Stevens R (2014) The SEED and the Rapid Annotation of microbial genomes using Subsystems Technology (RAST). Nucleic Acids Res. 42
(Database issue): D206-D214.

Waksman SA, Joffe JS (1922) Thiobacillus thiooxidans, a new sulfur-oxidizing organism isolated from the soil. J. Bacteriol. 7: 239-256.

Yarza P, Yilmaz P, Pruesse, E, Glöckner FO, Ludwig W, Schleifer KH, Whitman WB, Euzeby J, Amann R, Rossello-Mora R (2014) Uniting the classification of cultured and uncultured bacteria and archaea using 16S rRNA gene sequences. Nat. Rev. Microbiol. 12: 635-645.

Yoon SH, Ha SM, Lim JM, Kwon SJ, Chun J (2017). A large-scale evaluation of algorithms to calculate average nucleotide identity. Antonie Leeuwenhoek 110: 1281-1286. 